\section{PD-L1 and BCG response prediction}

A new study shows that tumours of patients with non-muscle-invasive bladder cancer (NMIBC) who do not respond to BCG therapy have elevated programmed cell death 1 ligand 1 (PD-L1) expression that is co-localized with $\mathrm{CD}^{+}$cells. The data show that intrinsic resistance to BCG might be caused by pretreatment adaptive immune responses and immune exhaustion.

Failure of intravesical BCG therapy occurs in $\sim 50 \%$ of patients, and multiple studies have been investigating possibilities to predict BCG response to enable early treatment with alternative agents. "In this study, we aimed to understand how immune checkpoint activation might mitigate BCG response and thereby affect response to immune checkpoint inhibitors in NMIBC," explains Max Kates from The Johns Hopkins School of Medicine, first author of the new paper. "We used tumour specimens before and after therapy from a large cohort of patients who underwent BCG treatment for NMIBC to understand how resistance is implicated in the mechanism of action of BCG." from 31 BCG responders and 32 BCG nonresponders of whom 13 relapsed and 5 progressed to muscle-invasive disease. Using immunohistochemical full slide review and a combined positivity score method, the researchers found PD-L1 expression in 0-4\% of responders but $25-28 \%$ of nonresponders $(P<0.01)$ before treatment. In all $\mathrm{PD}-\mathrm{L}^{+}{ }^{+}$samples, $\mathrm{PD}-\mathrm{L} 1^{+}$cells and $\mathrm{CD} 8^{+}$cells co-localized and the density of $\mathrm{CD}^{+}$cells was increased in $\mathrm{PD}-\mathrm{L}^{+}$areas. Furthermore, in nonresponders before BCG therapy, $\mathrm{CD} 4^{+}$cell counts were low in those who were $\mathrm{PD}-\mathrm{L1}^{+}(12 \%)$ and high
The cohort comprised samples

in those who were $\mathrm{PD}-\mathrm{L1}^{-}(50 \%$, $P<0.01)$. Comparison of samples from nonresponders before and after BCG therapy showed no changes in immune-associated gene expression, numbers of $\mathrm{CD}^{+}$or $\mathrm{FOXP}^{+}$cells and PD-L1 expression; however, $\mathrm{CD}^{+}$ cell counts were increased after BCG in samples from both responders and nonresponders $(P=0.017)$.

"We found that adaptive immune

intrinsic resistance to BCG might be caused by pretreatment adaptive immune responses and immune exhaustion 5

\title{
First total penis, scrotum and abdominal wall transplant
}

A team from John Hopkins have reported the first total penis, scrotum and partial abdominal wall transplantation on a male veteran who had sustained trauma from an improvised explosive device.

In the past, patients with traumatic genital injuries have undergone phalloplasty; however, the neophallus does not have inherent erectile function and formation of strictures and fistulae are a problem. Penile transplant is an alternative, particularly in men who do not have viable donor sites for phalloplasty, such as those who have been severely injured in combat. "We first implemented the transplant procedure in genotypic males aged 18-69 years who have lost $75 \%$ or more of their penis due to traumatic injury and had no other donor sites for a phalloplasty," corresponding author
Rick Redett tells Nature Reviews Urology. "These are men that really had no other reconstructive options."

The patient's injury involved bilateral above-knee leg amputation, bilateral traumatic orchiectomy and scrotal loss with a resultant $1.5-\mathrm{cm}$-long remnant of penile tissue and urethra. A surgical technique was especially developed for this patient as - although the inferior epigastric, iliac and femoral arteries were normal - the dorsal penile and cavernosal arteries were insufficient to support the transplant. Thus, the deep inferior epigastric arteries were used to revascularize the dorsal penile arteries and the graft, and the external pudendal arteries were used to supplement blood supply to the proximal shaft, groin, abdomen and scrotum. As the patient had substantial soft tissue defects, the graft was matched exactly to these areas and included skin and fat from the lower abdominal wall, the entire penis and scrotum, and tissue from the medial thighs. The first step was formation of the urethral anastomosis between the patient and donor tissue, which was performed over a Foley catheter. Next, the corporal and vascular anastomoses were performed, and finally the recipient and donor dorsal nerves were coapted. Full perfusion of the graft was obtained by end-to-side anastomosis of the left donor external pudendal artery and a segment of donor femoral artery to the recipient's femoral artery.

Over 1 year after the transplant, the recipient has near-normal erectile and orgasmic function and reports substantial improvements in pleasure scores with normal sensation to the penile shaft and tip. He is also able to urinate standing. The team report that testicular prostheses will be placed in the future.

Annette Fenner

ORIGINAL ARTICLE Redett, R. J. III et al. Total penis, scrotum, and lower abdominal wall transplantation. N. Engl.J.Med. 381, 1876-1878 (2019) 